\title{
How Should the Allocation of Resources Adjust TO THE BABY BUST?
}

\author{
DAVID DE LA CROIX \\ Université catholique de Louvain \\ GÉRALDINE MAHIEU \\ National Bank of Belgium \\ ALEXANDRA RILLAERS \\ Universidad de Alicante
}

\begin{abstract}
We analyze the impact of a drop in fertility on the optimal allocation of resources in an overlapping generations economy where old workers care about leisure. We also characterize optimal dynamics and study the decentralization of the optimum by means of intergenerational transfers and/or public debt. We conclude that the policy recommendations of postponing retirement is fragile and depend on preferences and technologies. Also, even when the optimal adjustment of public debt goes into the expected direction in the long run-i.e., public debt should decrease-this may not be the case during the transition.
\end{abstract}

\section{Introduction}

Over the next half century, the demographic structure of most of the OECD countries is expected to change significantly due to the declining fertility observed since the end of the 1960s. Although opinions differ about the extent

David de la Croix, FNRS, CORE and IRES, Université catholique de Louvain, B-1348 Louvain-la-Neuve, Belgium (delacroix@ires.ucl.ac.be). Géraldine Mahieu, National Bank of Belgium, 1000 Brussels, Belgium (geraldine.mahieu@nbb.be). Alexandra Rillaers, Departamento de Fundamentos del Análisis Económico, Universidad de Alicante, 03071 Alicante, Spain (rillaers@merlin.fae.ua.es).

The financial support of the PAI program P5/10 and the ARC program "Growth and Incentive Design" is gratefully acknowledged. We are thankful to the participants to the T2M meeting, SED 2000 and to A. d'Autume, C. Azariadis, P. Dehez, R. Farmer, O. Galor, L. Gevers, T. Kehoe, M. Marchand, P. Michel, O. Paddison, P. Pestieau, V. Rios-Rull, H. Sneessens, two anonymous referees and the editor for their comments on an earlier draft.

Received March 2002; Accepted March 2003.

(C) 2004 Blackwell Publishing, Inc.

Journal of Public Economic Theory, 6 (4), 2004, pp. 607-636. 
of the problem, it is a general consensus that some adjustment is necessary. Most of the literature concerned with the aging of the population adopts a numerical approach. These papers provide evidence on the consequences of the demographic change and estimate the impact of various social security reforms (lowering pensions benefits, increasing contribution, reducing national debt in advance, postponing retirement). However, these empirical contributions are not very conclusive relative to the optimal strategy to follow, as none of them proposes a clear and precise choice criteria to rank the various policy options. Some study the effects on the macroeconomic outcomes (output, savings, taxation level, etc.), others on the actuarial fairness (ratio of present value of lifetime benefits over present value of lifetime contributions), or on utilities and welfare.

Our objective is to lead the debate from the design of parametric reforms to the optimal allocation of resources (consumption, leisure, and capital). The basic idea is that by looking at an optimum problem without looking first at formal policies, one can get some mileage on policy design. Endowed with the well-defined criterion of Ramsey (1928), we theoretically assess the impact of a fertility drop on the optimal allocation and determine the required policy reaction.

In contrast with the theoretical contributions in this field, our model includes endogenous retirement decisions and is therefore suitable to assess the respective merits of all policy options mentioned above. Various contributions analyze the capacity of intergenerational transfers to induce optimal allocations, ${ }^{1}$ but all these papers assume an exogenous retirement age. Endogenous retirement decisions are analyzed by $\mathrm{Hu}$ (1979) and Michel and Pestieau (1999). Hu (1979) assumes that workers optimally select the share of their time in the second period of life devoted to retirement. If the transfers are tied to the individual retirement decision, the pension system introduces some distortions on the labor-supply choice. Michel and Pestieau (1999) compare the decentralized equilibrium with the Golden Rule and show that in order to achieve the steady-state first-best optimum, one needs to control both an unrestricted pay-as-you-go transfer and the retirement age.

The short-term perspective is a critical issue in this debate since the new steady state may be far away from the initial one and may take long to reach. In addition to determining the effect of a reduced fertility on the first-best longrun allocation of resources, our paper also analyzes the optimal dynamics. We provide insights concerning the effects of the decline in fertility on the welfare

\footnotetext{
${ }^{1}$ Marchand, Michel and Pestieau (1996) study the divergence between the market and the optimal solutions in an overlapping generations model with changing productivity and fertility (in an endogenous growth setting). Boadway, Marchand and Pestieau (1991), Blanchet and Kessler (1991), Peters (1991), and Meijdam and Verbon (1997) also study the capacity of a pay-as-you-go social security system to reallocate resources across generations when fertility and productivity vary over time.
} 
of the first generations and show that they crucially depend on households' preferences.

This paper is organized as follows. Section 2 details the model economy and characterizes the problem of a benevolent planner. In Section 3, we analyze the long-run effect of a drop in fertility. On one hand, we find that except when parameters of production and utility function take extreme values, the steady-state capital stock per old worker will be higher after the reduction in fertility. On the other hand, the optimal adjustment of the retirement age crucially depends on the characteristics of the production technology and of the preferences of the agents. Section 4 deals with the policies that should be undertaken to cope with the drop in fertility. This is done by the way of decentralization theorems. Section 5 focuses on the dynamics along the transition path to the new steady state and highlights the short-run effect of the demographic shock on both the optimal allocation of resources and on the optimal debt and transfers policies. We show that even when optimal public debt goes into the expected direction in the long run, this is not necessarily true during the transition. Section 6 concludes.

\section{The Planner's Problem}

We consider an overlapping generations economy à la Diamond (1965) with one physical good that can be either consumed or stored in the form of capital. Time is discrete and goes from zero to infinity. The households live two periods, they consume and work during these two periods. They supply inelastically one unit of labor when young. When old they get utility from leisure and they work a share $\lambda$ of their time endowment, as in $\mathrm{Hu}$ (1979). This share $\lambda$ belongs to $(0,1)$. The choice of $\lambda$ therefore determines the retirement age. The assumed difference in the elasticity of labor supply over the life cycle is motivated by several key facts on labor supply and retirement behavior, summarized in Rust and Phelan (1997).

Households have a utility function defined over consumption when young, consumption when old, and leisure when old. It is separable and takes the following form:

$$
U(\cdot)=u\left(c_{t}\right)+\beta u\left(d_{t+1}\right)-v\left(\lambda_{t+1}\right),
$$

where $\beta \in(0,1)$ is the psychological discount factor. The instantaneous utility $u(\cdot)$ is a strictly increasing, concave function from $\mathbb{R}_{+}$to $\mathbb{R}$. The derivatives are denoted $u_{c}>0$ and $u_{c c}<0$. It is smooth on the interior of $\mathbb{R}_{+}$and

$$
\lim _{c \rightarrow 0} u_{c}(c)=+\infty
$$

The disutility of work $v(\cdot)$ is a strictly increasing, convex function from $[0,1]$ to $\mathbb{R}$. It is smooth on $(0,1)$ and

$$
\lim _{\lambda \rightarrow 0} v_{\lambda}(\lambda)=0 \quad \text { and } \quad \lim _{\lambda \rightarrow 1} v_{\lambda}(\lambda)=+\infty
$$


The population grows exogenously with

$$
n_{t}=\frac{N_{t}}{N_{t-1}}>0 .
$$

The variable $n_{t}$ denotes the growth factor of the population. In our settings, the demographic change is a once-for-all reduction in $n_{t}$. This corresponds to a drop in the fertility rate at time $t$ : at time $t$, young workers represent a smaller fraction of total population.

Firms have a production function displaying constant returns to scale: $f\left(K_{t}, L_{t}\right)$. It is an increasing and concave function from $\mathbb{R}_{+}^{2}$ to $\mathbb{R}_{+}$, homogeneous of degree 1 . It is smooth on the interior of $\mathbb{R}_{+}$. The derivatives are denoted $f_{k}, f_{l}>0, f_{l l}, f_{k k}<0$, and $f_{k l}>0$. When the labor input $L_{t}$ equals $N_{t}+\lambda_{t} N_{t-1}$, production is given $\mathrm{by}^{2}$

$$
f\left(K_{t}, N_{t}+\lambda_{t} N_{t-1}\right) .
$$

Denoting the capital stock per old worker $k_{t}=K_{t} / N_{t-1}$, output per old worker is given by $y_{t}=f\left(k_{t}, n_{t}+\lambda_{t}\right)$. The resource constraint of the economy is

$$
f\left(k_{t}, n_{t}+\lambda_{t}\right)=n_{t} k_{t+1}+n_{t} c_{t}+d_{t} .
$$

The capital intensity is denoted as

$$
\tilde{k}_{t}=\frac{K_{t}}{N_{t}+\lambda_{t} N_{t-1}}=\frac{k_{t}}{n_{t}+\lambda_{t}} .
$$

The question of the choice of the planner's discount factor is an old debate. Michel (1990b) argues that, within an utilitarian setup, one should choose the discount rate, which allows the economy to converge to the Golden Rule. This discount rate is equal to the growth rate of population, and the corresponding social objective function is the undiscounted sum of Ramsey. According to Ramsey (1928) "it is assumed that we do not discount later enjoyments in comparison with earlier ones, a practice which is ethically indefensible and arises merely from the weakness of imagination," thus the social planner maximizes the undiscounted sum of the life-cycle utility of a

\footnotetext{
${ }^{2}$ Allowing for different levels of productivity of young and old workers would only require a slight modification of the production function. This would be given by $f\left(K_{t}, N_{t}+\lambda_{t} N_{t-1} \theta\right)$ with $\theta<1(\theta>1)$ implying a negative (positive) return to seniority. It is further assumed for tractability that young workers and old workers are perfect substitutes. Removing this assumption requires the use of a production function with three inputs, as in Crettez and Le Maitre (2002); they assume that total labor input is a nonlinear combination of old and young workers $L_{t}=G\left(N_{t}, \lambda_{t} N_{t-1}\right)$ and focus their attention on the elasticity of substitution between the two types of workers. To perform this study they need to simplify other parts of the model, by assuming logarithmic utility and Cobb-Douglas production.
} 
representative individual of all current and future generations. As this social welfare objective may not be defined, we consider, as Ramsey did,

$$
\sum_{t=-1}^{\infty}\left(U\left(c_{t}, d_{t+1}, \lambda_{t+1}\right)-\hat{U}\right)
$$

with $k_{0}$, and $c_{-1}$ given. Here $c_{-1}$ is the hypothetical youth consumption of the first old generation. $\hat{U}=\sup \{U(c, d, \lambda)$ under the resource constraint $\}$ is the maximum stationary utility. Ramsey assumes that this maximum utility is finite, as "economic causes alone could never give us more than a certain finite rate of enjoyment." In our model, this is the case if the capital that maximizes the long-term net production is finite. This is true if the following assumption is satisfied.

Assumption 1:

$$
\forall n \quad \lim _{k \rightarrow 0} f_{k}(k, n)>n>\lim _{k \rightarrow+\infty} f_{k}(k, n+1) .
$$

Alternative social welfare functions include a Benthamite function with discounting and a Rawlsian maximin function. In the first case, we have to assume an exogenous discount factor smaller than $1 / n$ to ensure the convergence of the infinite sum. The problem with this approach is that the ex ante choice of the constant discount factor is equivalent to an ex post choice of the long-run stationary state (the so-called modified Golden Rule). As far as the second case is concerned, see the application of the maximin rule in a growth framework by Solow (1974).

As the life-cycle utility function is separable, we can rearrange the objective function in the following way (grouping the contemporaneous terms together and ignoring the constant term $\left.u\left(c_{-1}\right)\right)$ :

$$
\mathcal{W}=\sum_{t=0}^{\infty}\left[u\left(c_{t}\right)+\beta u\left(d_{t}\right)-v\left(\lambda_{t}\right)-\hat{U}\right]
$$

The planner thus maximizes $\mathcal{W}$ given an initial capital stock $k_{0}$ and given the resource constraint (2).

DeFINITION 1 (Optimal allocation): Given an exogenous path $\left(n_{t}\right)_{t \geq 0}$ and an initial capital $k_{0}>0$, an optimal allocation is a sequence of strictly positive quantities $\left(c_{t}, d_{t}, \lambda_{t}, k_{t+1}\right)_{t \geq 0}$ with $\lambda_{t} \in(0,1)$ such that the objective function (3) is maximized subject to the resource constraint (2).

The Lagrangean $\mathcal{L}_{t}$ for period $t$ is the sum of the current utilities and of the increase in the shadow value of the capital stock, $q_{t+1} k_{t+1}-q_{t} k_{t}$, i.e., 


$$
\mathcal{L}_{t}=u\left(c_{t}\right)+\beta u\left(d_{t}\right)-v\left(\lambda_{t}\right)+\frac{q_{t+1}}{n_{t}}\left(f\left(k_{t}, n_{t}+\lambda_{t}\right)-n_{t} c_{t}-d_{t}\right)-q_{t} k_{t}
$$

For an interior optimal solution, the derivatives of $\mathcal{L}_{t}$ with respect to $c_{t}, d_{t}, \lambda_{t}$, and $k_{t}$ must be equal to zero. Hence,

$$
\begin{aligned}
u_{c}\left(c_{t}\right) & =q_{t+1} \\
u_{c}\left(d_{t}\right) & =\frac{q_{t+1}}{\beta n_{t}} \\
v_{\lambda}\left(\lambda_{t}\right) & =\frac{q_{t+1}}{n_{t}} f_{l}\left(k_{t}, n_{t}+\lambda_{t}\right) \\
\frac{q_{t+1}}{n_{t}} & =\frac{q_{t}}{f_{k}\left(k_{t}, n_{t}+\lambda_{t}\right)} .
\end{aligned}
$$

An optimal path $\left(c_{t}, d_{t}, q_{t}, \lambda_{t}, k_{t+1}\right)_{t \geq 0}$ satisfies $(4)-(7)$ and the transversality condition. $^{3}$

\section{Long-Term Effect of the Baby Bust}

Consider that the growth factor of the population is constant, i.e., $n_{t}=n \forall t$. Then, a steady state is a stationary path $\left(c_{t}, d_{t}, q_{t}, \lambda_{t}, k_{t+1}\right)=(c, d, q, \lambda, k)$ with positive quantities verifying the optimality conditions:

$$
\begin{aligned}
u_{c}(c) & =q \\
u_{c}(d) & =\frac{q}{\beta n} \\
v_{\lambda}(\lambda) & =\frac{q}{n} f_{l}(k, n+\lambda) \\
f_{k}(k, n+\lambda) & =n \\
f(k, n+\lambda) & =n k+n c+d .
\end{aligned}
$$

PROPOSITION 1 (Existence and uniqueness of the optimal solution): Under Assumption 1, the steady state of the dynamics described by (4)-(7) is characterized by the Golden Rule (11) and it is stable in the saddle-point sense.

See Appendix A for a proof. Note that the assumption made in Proposition 1 is weaker than the usual Inada conditions. ${ }^{4}$

\footnotetext{
${ }^{3}$ The transversality condition of this problem states that the limit of the capital stock is the Golden Rule capital stock in the case where it exists (see Michel (1990a)).

${ }^{4}$ This will allow us to provide examples with CES production functions.
} 


\subsection{Optimal Capital}

PROPOSITION 2 (Population growth and optimal capital): The optimal capital intensity increases when fertility drops: $\frac{d \tilde{k}}{d n}<0$. With a CES production function,

$f(k, l)=A\left[\alpha k^{-\rho}+(1-\alpha) l^{-\rho}\right]^{-1 / \rho}, \quad A \in \mathbb{R}_{+}, \quad \alpha \in(0,1), \quad \rho \in(-1, \infty) \backslash\{0\}$, the capital per old workers also increases iffactors are low substitutes: $\frac{d k}{d n}<0$ if $\rho \leq 0$.

See Appendix B for a proof. The decrease in fertility, and therefore in labor supply is accompanied by a rise in capital intensity: labor is being replaced by capital, the extent of which is positively related to the degree of substitutability between both production factors.

With a CES production function, the parameter $\rho$ determines the degree of substitutability between the production factors. The smaller $\rho$, the larger the degree of substitutability, and the more a decrease in fertility and thereby in labor supply will be accompanied by a rise in capital per old worker: labor is replaced by capital: $d k / d n<0$ (note that Proposition 2 applies to a CobbDouglas production function $(\rho \rightarrow 0))$.

When capital and labor are poor substitutes (when $\rho$ is large) however, a decrease in $n$ might be accompanied by a decrease in $k$ :

COROLLARY 1: There exist $\bar{\rho}, \bar{\sigma}$ such that for all $\rho>\bar{\rho}$ and all $\sigma<\bar{\sigma}$ we have $d k / d n>0$.

See Appendix $\mathrm{C}$ for a proof. When $\rho$ is very large, a decrease in $n$ is likely to be accompanied by a decrease in $k(d k / d n>0)$. The opposite result may only arise if labor input has risen, which implies that $\lambda$ has increased so much that it outweighs the drop in $n$. This only occurs if agents are ready to substitute consumption for leisure, which is the case when $\sigma$ is large. At given $v$, when $\sigma$ is small, households do not accept consumption as a substitute for leisure and so $k$ will move in the same direction as $n$.

\subsection{Optimal Consumption Profile}

The drop in fertility further affects the optimal allocation of consumption over the life cycle. This is closely linked with the adjustment of $q$, as stated in the following proposition:

PROPOSITION 3:

$$
\text { If } k+c-f_{l}>0, \quad \text { then } \frac{d q}{d n}>0 .
$$

In this case, the fertility drop increases young-age consumption:

$$
\frac{d c}{d n}=-c \sigma(c) \frac{d q}{d n} \frac{1}{q}<0
$$


while the effects on old-age consumption is given by

$$
\frac{d d}{d n}=d \sigma(d)\left(\frac{1}{n}-\frac{d q}{d n} \frac{1}{q}\right),
$$

where $\sigma(\cdot)$ is the elasticity of substitution between consumption at two points of time.

See Appendix D for a proof. The expression $k+c-f_{l}>0$ represents the derivative of the resources constraint with respect to $n$. Given the optimal capital stock, this constraint represents the feasible combinations of $d, c$, and $\lambda$. When the above condition is satisfied, the drop in $n$ relaxes the resources constraint: the reduction of resources necessary to maintain consumption of the young and capital per old worker ${ }^{5}$ overcomes the consecutive decrease in total output caused by the drop in labor supply.

The expression $k+c-f_{l}>0$ can also be interpreted in the light of the quest for the optimal growth rate of population by Samuelson (1975), Deardoff (1976), and Samuelson (1976). The objective of Samuelson (1975) is to maximize steady-state social welfare by choosing both the allocation of resources and the growth rate of population $n$. It appeared in the subsequent debate Deardoff (1976)-Samuelson (1976) that the objective is rarely concave with respect to $n$ and that its slope is likely to be negative. This is in conformity with the old result of neoclassical growth theory that economies benefit from low or negative population growth. In our setup with labor supply of old workers, the problem of the optimal growth rate of population amounts to maximize the following Lagrangean:

$$
\max _{c, d, k, \lambda, n} u(c)+\beta u(d)-v(\lambda)+\theta(f(k, n+\lambda)-n(c+k)-d) .
$$

The term $\theta\left(f_{l}-k-c\right)$ is the derivative with respect to $n$. The condition $k+c-f_{l}>0$ is thus equivalent to assume that the objective is decreasing in $n$. In the sequel we follow Deardoff (1976) and Samuelson (1976) and consider that $k+c-f_{l}>0$, although we occasionally provide the results under the opposite assumption.

When $k+c-f_{l}>0$, the drop in fertility constitutes a positive shock, which releases resources in the economy. These freed resources can potentially be allocated to consumption of the young and of the old, and to leisure. The following corollary indicates a priority ranking in the optimal allocation of the freed resources: young-age consumption is given priority, then comes the consumption when old, and finally, the retirement age is eventually decreased.

COROLLARY 2:

$$
\frac{d \lambda}{d n}>0 \Rightarrow \frac{d d}{d n}<0 \Rightarrow \frac{d c}{d n}<0 .
$$

${ }^{5}$ The respectively so-called "intergenerational effect" and "capital thickening effect"; see Cutler et al. (1990) and Meijdam and Verbon (1997). 
See Appendix E for a proof. Resources will first of all be used to increase the consumption of the young as it is relatively less expensive than the consumption of the old when fertility has dropped. The planner may further decide to either increase or decrease the consumption of the old and the retirement age depending on the shape of the utility function. In the next subsections, we shall focus on the role of $\sigma$. Indeed, at given $v, \sigma$ determines the extent to which the three commodities are substitutable in the utility function.

\subsection{Optimal Retirement Age}

We use the total derivative of Equation (11) in order to compute $d \lambda / d n$ :

PROPOSITION 4 (Population growth and optimal retirement age): The effect of $a$ drop in fertility on the optimal retirement age at the steady state is given by

$$
\frac{d \lambda}{d n}=\left(-1+\frac{1}{f_{k l}}\right)+\frac{n+\lambda}{n} \frac{d k}{d n} \frac{n}{k^{\prime}} .
$$

The total effect of a fertility drop can be decomposed in two different effects. The first term in (13) constitutes the direct effect of a change in $n$, while the second term represents the effect induced by a change in $n$ through a subsequent change in $k$.

The intuition behind the direct effect can be understood by looking at the Golden Rule (Equation (11)). When calculating its total derivative (holding $k$ constant) we obtain $f_{k l}(d n+d \lambda)=d n$ : a drop in $n$ (a) reduces the optimal marginal productivity of capital per old worker, and (b) effectively decreases the marginal productivity of capital per old worker by diminishing labor input. The importance of this second effect is determined by $f_{k l}$. When dividing the total derivative by $d n$ and by $f_{k l}$, we have $d \lambda / d n=1 / f_{k l}-1$. This expression, which corresponds to the first term in (13), indicates by how much the retirement age should change following a drop in $n$ (and holding $k$ constant), in order to stick to the Golden Rule. If $f_{k l}=1$ and if a change in $n$ is not accompanied by a change in $k(d k / d n=0)$, the retirement age should remain unchanged.

However, a change in $n$ will also induce a change in steady-state capital per old worker. The second term in (13) depends on the elasticity of capital per old to population growth. The effect on $\lambda$ of a change in $n$ through this elasticity always goes in the same direction as $d k / d n$. Indeed, given that the marginal productivity of labor rises with capital, an increase (decrease) in capital input gives an incentive (for the planner) to increase (decrease) the labor input through a rise (drop) in the retirement age. The relative importance of these two effects will finally determine the sign of $d \lambda / d n$. The value of $\sigma$ plays an important role in the balance of these two effects.

COROLLARY 3: There exists $\hat{\sigma}$ such that for all $\sigma>\hat{\sigma}$ we have $d \lambda / d n<0$. 
See Appendix $\mathrm{F}$ for a proof. As mentioned before, a large $\sigma$ given $v$ implies that agents easily substitute consumption for leisure. They are thus ready to give up leisure in order to promote young-age consumption when fertility declines.

Finally, note that the optimal adjustment of the retirement age to a fertility drop is ambiguous only in the case the resources constraint is relaxed:

COROLLARY 4: When a drop in fertility tightens the resources constraint, i.e., $k+$ $c-f_{l}<0$, the optimal retirement age should increase $(d \lambda / d n<0)$.

See Appendix F for proof.

\section{Optimal Policy}

Various empirical evaluations have been carried out to highlight the impact of demographic change and the effect of potential reforms. Some authors have estimated the effect of adjusting intergenerational transfers through an increase in contribution rates, a reduction of pension benefits, or a switch to a fully funded scheme. Examples are in Miles (1999), Chauveau and Loufir (1996), kotlikoff (1996), and Conesa and Krueger (1999). Other studies have considered an additional reform: a change in the retirement age. Examples are Cazes et al. (1994), Auerback et al. (1989), De Nardi, Imrohoroglu, and Sargent (1999), and Kotlikoff, Smelters, and Walliser (2001). The reduction in national debt is considered as a potential reform by some economists, as Huang, Imrohoroglu, and Sargent (1997) or Greenspan. ${ }^{6}$

Having characterized the evolution of the optimal allocation of resources following a reduction of fertility, we are now able to compute the optimal policy reaction using decentralization theorems. Assuming both that an optimum policy exists and that it converges to a steady state, Atkinson and Sandmo (1980) show that a first-best allocation can be achieved if the government can use lump-sum taxes that redistribute wealth among generations. As an alternative to those transfers, Diamond (1965) shows that public debt can be used as an instrument to decentralize the Golden Rule as a competitive equilibrium. We now extend these setups to our model.

\subsection{Decentralization with Lump-Sum Transfers}

In this section, we assume that the government provides the young and the old with a lump-sum transfer. We characterize the competitive equilibrium with lump-sum transfers. The maximization program of the individual is

$$
\max _{c_{t}, d_{t+1}, \lambda_{t+1}} u\left(c_{t}\right)+\beta u\left(d_{t+1}\right)-v\left(\lambda_{t+1}\right)
$$

\footnotetext{
${ }^{6}$ As Greenspan advocated in March 2000 in testimony to the Congressional Special Committee on Aging, using budget surpluses to repay national debt would be the best for the U.S. economy and a good way to prepare for the baby boomers' retirement.
} 


$$
\begin{aligned}
\text { subject to } \quad c_{t}+s_{t} & =w_{t}+\tau_{t}^{1} \\
d_{t+1} & =R_{t+1} s_{t}+\tau_{t+1}^{2}+w_{t+1} \lambda_{t+1},
\end{aligned}
$$

where $\tau_{t}^{1}, \tau_{t+1}^{2} \in \mathbb{R}$ are lump-sum transfers, $s_{t}$ represents savings, and $R_{t+1}$ is the interest factor. $w_{t}$ and $w_{t+1}$ denote wages. The maximization problem has a solution if the life-cycle income is positive. The first-order conditions are

$$
u_{c}\left(c_{t}\right)=\beta R_{t+1} u_{c}\left(d_{t+1}\right)
$$

and

$$
\beta u_{c}\left(d_{t+1}\right) w_{t+1}=v_{\lambda}\left(\lambda_{t+1}\right) .
$$

The first condition allows to define a saving function

$$
s_{t}=s\left(w_{t}+\tau_{t}^{1}, \tau_{t+1}^{2}+w_{t+1} \lambda_{t+1}, R_{t+1}\right),
$$

with its derivatives satisfying $s_{1} \in(0,1), s_{2} \in(-1,0)$, and $s_{3} \in \mathbb{R}$.

The competitive behavior of firms leads to the equalization of marginal productivities to marginal costs:

$$
\begin{gathered}
R_{t}=f_{k}\left(k_{t}, n_{t}+\lambda_{t}\right) \\
w_{t}=f_{l}\left(k_{t}, n_{t}+\lambda_{t}\right) .
\end{gathered}
$$

The budget constraint of the government is

$$
\tau_{t}^{2}=-\tau_{t}^{1} n_{t},
$$

and the equilibrium condition in the capital market implies

$$
k_{t+1}=s\left(w_{t}+\tau_{t}^{1}, \tau_{t+1}^{2}+w_{t+1} \lambda_{t+1}, R_{t+1}\right) .
$$

Definition 2 (Competitive equilibrium with lump-sum transfers): Assume an exogenous path $\left(n_{t}\right)_{t>0}$, an initial capital $k_{0}>0$, and a transfer system $\left(\tau_{t}^{1}, \tau_{t}^{2}\right)_{t \geq 0}$ satisfying (19). A competitive, perfect-foresight, intertemporal equilibrium is a vector $\left(c_{t}, d_{t}, \lambda_{t}, k_{t}, s_{t}, R_{t}, w_{t}\right)_{t \geq 0}$ starting at $k_{0}$ and satisfying the conditions (14)-(18) and (20).

PROPOSITION 5 (Decentralization with lump-sum transfers): For any optimal allocation with positive quantities $\left(c_{t}^{\star}, d_{t}^{\star}, \lambda_{t}^{\star}, k_{t}^{\star}\right)_{t \geq 0}$ starting at $k_{0}$, there exists a transfer system $\left(\tau_{t}^{1}, \tau_{t}^{2}\right)_{t \geq 0}$ satisfying (19) such that this trajectory is an intertemporal equilibrium with perfect foresight. The sequences $\left(\tau_{t}^{1}, \tau_{t}^{2}\right)_{t \geq 0}$ satisfy

$$
\tau_{t}^{2}=-n_{t} \tau_{t}^{1}=n_{t}\left(f_{l}\left(k_{t}^{\star}, n_{t}+\lambda_{t}^{\star}\right)-k_{t+1}^{\star}-c_{t}^{\star}\right) .
$$

See Appendix G for a proof. Lump-sum transfers can be used to set the level of savings so as to obtain the optimal stock of capital. Since both 
the individual allocation rule of consumption over the life cycle and the labor-supply rule are the same as the optimal ones, there is no need for another instrument. This proposition generalizes the result of Michel and Pestieau (1999) to non-steady-state allocations and to the case where the retirement age is not mandatory. Observe that, when the fertility drop relaxes the resources constraint of the economy, the transfer from old workers to young workers is positive. Note that, at steady state, we have

$$
\tau^{1}=-\left(f_{l}\left(k^{\star}, n+\lambda^{\star}\right)-k^{\star}-c^{\star}\right)=-\frac{\tau^{2}}{n} .
$$

\subsection{Optimal Public Debt Policy}

When public debt is used and transfers are imposed on the young generation only (this is the Diamond (1965) setup), the individuals' budget constraints become

$$
\begin{aligned}
c_{t}+s_{t} & =w_{t}+\tau_{t} \\
d_{t+1} & =R_{t+1} s_{t}+w_{t+1} \lambda_{t+1},
\end{aligned}
$$

while the budget constraint of the government becomes

$$
R_{t} b_{t}+n_{t} \tau_{t}=n_{t} b_{t+1}
$$

where $b_{t} \equiv B_{t} / N_{t-1}$ is the debt per old worker. The debt is held by the households. It thus diverts part of private savings from productive capital:

$$
b_{t+1}+k_{t+1}=s\left(w_{t}+\tau_{t}, w_{t+1} \lambda_{t+1}, R_{t+1}\right) .
$$

Definition 3 (Competitive equilibrium with public debt): Assume an exogenous path $\left(n_{t}\right)_{t \geq 0}$, an initial capital $k_{0}>0$, and a debt policy $\left(b_{t}, \tau_{t}\right)_{t \geq 0}$ satisfying (24). A competitive, perfect-foresight, intertemporal equilibrium is a vector $\left(c_{t}, d_{t}, \lambda_{t}, k_{t}, s_{t}, R_{t}, w_{t}\right)_{t \geq 0}$ starting at $k_{0}$ and satisfying the conditions (16)(18) and (22)-(25).

PROPOSITION 6 (Decentralization with public debt): For any optimal allocation with positive quantities $\left(c_{t}^{\star}, d_{t}^{\star}, \lambda_{t}^{\star}, k_{t}^{\star}\right)_{t \geq 0}$ starting at $k_{0}$ there exists a debt policy $\left(b_{t+1}, \tau_{t}\right)_{t \geq 0}$ such that this trajectory is an intertemporal equilibrium with perfect foresight. The sequence $\left(b_{t+1}, \tau_{t}\right)_{t \geq 0}$ satisfies

$$
\begin{aligned}
b_{t+1} & =f_{l}\left(k_{t}^{\star}, n_{t}+\lambda_{t}^{\star}\right)-k_{t+1}^{\star}-c_{t}^{\star}+\tau_{t} \\
\tau_{t} & =\frac{n_{t} b_{t+1}-f_{k}\left(k_{t}^{\star}, n_{t}+\lambda_{t}^{\star}\right) b_{t}}{n_{t}},
\end{aligned}
$$

$b_{0}$ being given. 
See Appendix $\mathrm{H}$ for a proof. Debt ${ }^{7}$ can be used to obtain the optimal stock of capital and lump-sum transfers to the young balance the budget or vice versa. Note that, at steady state, the optimal transfer is zero since the Golden Rule $f_{k}=n$ holds

$$
\begin{aligned}
& b=f_{l}\left(k^{\star}, n+\lambda^{\star}\right)-k^{\star}-c^{\star} \\
& \tau=0 .
\end{aligned}
$$

\subsection{Optimal Policy Response}

In the long run, the effect of a demographic change on the optimal transfer to the young or on the level of debt is given by the following proposition.

PROPOSITION 7 (Optimal policy response):

$$
-\frac{d \tau^{1}}{d n}=\frac{d b}{d n}>0 \Leftrightarrow-k \frac{d k}{d n} \frac{n}{k}-c \frac{d c}{d n} \frac{n}{c}-\frac{k}{n+\lambda}>0 .
$$

See Appendix I for a proof. If optimal capital per old worker increases sufficiently, and if it is optimal to sufficiently increase the consumption of the young, then one should reduce public debt or alternatively one should favor transfers to the young.

Given that $\tau^{2}=-n \tau^{1}=n b$, the effect of a fertility drop on the transfers to the old is given by $n d b / d n+b$ with $b=f_{l}-c-k$. This allows us to state the following:

PROPOSITION 8 (Comparison of policy instruments):

$$
\begin{gathered}
\text { If } k+c-f_{l}>0, \quad \text { then } \frac{d \tau^{2}}{d n}>0 \Rightarrow \frac{d b}{d n}>0 \quad \text { and } \frac{d b}{d n}>0 \nRightarrow \frac{d \tau^{2}}{d n}>0, \\
\text { If } k+c-f_{l}<0, \quad \text { then } \frac{d b}{d n}>0 \Rightarrow \frac{d \tau^{2}}{d n}>0 \quad \text { and } \frac{d \tau^{2}}{d n}>0 \nRightarrow \frac{d b}{d n}>0 .
\end{gathered}
$$

The assumption $k+c-f_{l}>0\left(k+c-f_{l}<0\right)$ implies that the resource constraint becomes less (more) stringent after the drop in fertility. When $k+c-f_{l}>0\left(k+c-f_{l}<0\right)$, reducing public debt is more (less) robust a policy response than adjusting intergenerational transfers.

\section{Optimal Dynamics with an Anticipated Baby Bust}

The previous sections focused on the long-run optimal adjustment to the baby bust. However, the new steady state may be far away from the initial one and

\footnotetext{
${ }^{7} \mathrm{~A}$ negative debt is not excluded and corresponds to a situation where the government detains a part of the productive capital. This will be the case if the drop in $n$ releases resources at the optimal steady state.
} 


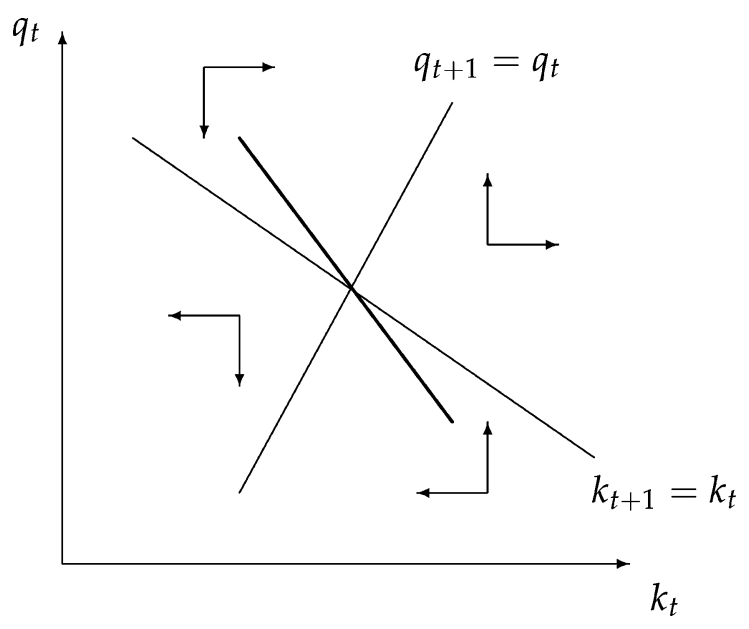

Figure 1: The phase diagram

long to reach. Until so far we did not provide insights concerning the effects of this shock during the transition and hence on the welfare of the first generations, which actually matter in the current policy debate. In the present section, we study the optimal dynamics. For the sake of reality, the baby bust is supposed to be anticipated by one period. Indeed, in this theoretical framework individuals are only represented when they have reached the working age. It is therefore realistic to assume that the shock affecting the economy in period 2 is observed and anticipated today (period 1).

The dynamics of the system around the steady state can be described using the phase diagram in Figure 1. A detailed derivation of the phaselines and the direction of motion can be found in Appendix J. The intersection of the two phaselines represents the steady state. This figure illustrates that there is only one trajectory converging to the steady state (represented by the bold line in Figure 1).

This graphic can be used to study the dynamic adjustment following a drop in fertility. In the light of the previous results, we focus on the case where the drop in $n$ relaxes the resources constraint (implying $d q / d n>0$ ) and increases the optimal capital stock per old $(d k / d n<0)$. In this situation, the phaseline $q_{t+1}=q_{t}$ shifts unambiguously to the right. The phaseline $k_{t+1}=k_{t}$ may shift either upward or downward, when the elasticity of substitution $\sigma$ is respectively large and small.

Figure 2 displays the dynamic adjustment associated with the two cases. The solid and dotted lines represent the phaselines before and after the shock, respectively. Bold dots represent the optimal path. Time 0 is the initial steady state. At time 1 the shock is anticipated and it takes place at time 2 . The thin arrow indicates the new saddle path. A planner anticipating (in period 1) a fertility drop for the next period (period 2) will change the allocation in 

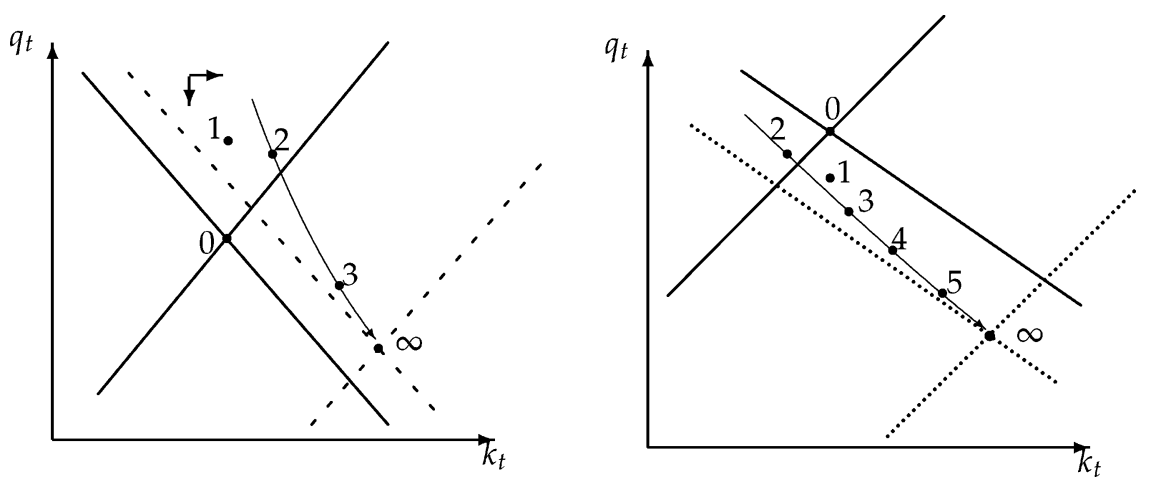

Figure 2: Dynamics: cases 1 and 2

period 1 so as to be on the new saddle path in the next period. As the line $k_{t+1}=k_{t}$ shifts upward in case 1 and downward in case 2 , the saddle path is located above the initial line $k_{t+1}=k_{t}$ in case 1 and below in case 2 .

In case $1, q$ is higher than its initial value in period 1 and 2 . Hence, in period 1 ( $n$ still unchanged and $k$ still at the initial steady state), $c$ and $d$ must be decreased. This consumption squeeze fosters capital accumulation and allows for a rapid convergence to the higher long-term capital stock. Consequently, not only current old individuals but also currently young individuals will bear the cost of the rapid transition to the new steady state to the benefit of all future generations. The story is different in case 2. The planner uses the capital freed by the "capital-thickening effect" to increase young-age and oldage consumptions in period 1 (reflected by the drop in $q$ ). This temporarily reduces the capital accumulation and puts the economy on the new saddle path. Along this path, the adjustment is slower than in the first case, but no generation is loosing.

Example: Let us numerically illustrate the optimal transition in the centralized economy, and analyze how this translates into optimal dynamics of debt and intergenerational transfers. We assume a utility function with constant elasticity of substitution:

$$
u(c)=\left(1-\sigma^{-1}\right)^{-1} c^{1-1 / \sigma}, \quad \sigma \in(0, \infty) \backslash\{1\}
$$

and the following disutility:

$$
v(\lambda)=-\frac{\ln (1-\lambda)+\lambda}{2}
$$

which satisfies $v_{\lambda}(0)=0$ and $v_{\lambda}(1)=\infty$. We assume a Cobb-Douglas production function: $f(k, n+\lambda)=A k^{\alpha}(n+\lambda)^{1-\alpha}$. We choose a psychological discount factor of $4 \%$ per year $(\beta=0.2727)$, a share of capital income in output of $0.3(\alpha=0.3)$, and $A=2.5$. The parameter $n$ captures both the demographic growth rate and the rate of improvement 
Table 1: Dynamic effects of the baby bust on the optimal allocation

\begin{tabular}{lccccccr}
\hline & \multicolumn{3}{c}{$\boldsymbol{\sigma}=\mathbf{2}$} & & \multicolumn{3}{c}{$\boldsymbol{\sigma}=\mathbf{0 . 1}$} \\
\cline { 2 - 4 } \cline { 6 - 8 } $\boldsymbol{t}$ & $\boldsymbol{c}_{\boldsymbol{t}}$ & $\boldsymbol{d}_{\boldsymbol{t}}$ & $\mathbf{1}-\boldsymbol{\lambda}_{\boldsymbol{t}}$ & & $\boldsymbol{c}_{\boldsymbol{t}}$ & $\boldsymbol{d}_{\boldsymbol{t}}$ & $\mathbf{1}-\boldsymbol{\lambda}_{\boldsymbol{t}}$ \\
\hline 1 & -1.0 & -1.0 & -0.2 & & +1.4 & +1.4 & +8.1 \\
2 & +10.0 & -17.8 & -6.3 & & +2.4 & +0.9 & +4.4 \\
3 & +12.4 & -16.1 & -6.7 & & +3.0 & +1.5 & +7.0 \\
4 & +12.9 & -15.7 & -6.8 & & +3.4 & +1.9 & +8.5 \\
$\infty$ & +13.0 & -15.6 & -6.8 & & +3.9 & +2.4 & +10.7 \\
\hline
\end{tabular}

Note: Percentage deviation from a constant $n$ solution.

in the efficiency of the labor force (exogenous technological progress). The demographic shock is modeled as a permanent drop in $n$ from $3 \%$ per year to $2.5 \%$, which implies that $n$ goes down from 2.43 to 2.10 .

Table 1 illustrates the dynamic effects in terms of resources allocation in our example. Case 1 is illustrated by the example with $\sigma=2$ (columns $1-3)$. The cost supported by the first old and the first young generations appears clearly under the form of a drop in their consumption levels and an increase in the retirement age. Case 2 is illustrated by the example with $\sigma=$ 0.1 (columns 4-6). In this case, the consumption and leisure of all generations are increased. So no generation loses in the transition process.

The analysis of the optimal dynamics highlights that the welfare effects of a fertility drop on the initial generations again crucially depend on the parameters, and in particular on the concavity of the utility function. Table 2 displays the corresponding optimal policy reactions as far as the decentralization instruments are concerned.

We observe that the sign of the long-run optimal adjustment in terms of public debt and transfers to the young is insensitive to the concavity of the utility function: the increase in transfers to the young workers and the reduction of national debt constitute an optimal long-run response in both cases $(\sigma=2$ and $\sigma=0.1)$.

Table 2: Dynamic effects of the baby bust on optimal transfers and debt

\begin{tabular}{lccccccc}
\hline & \multicolumn{3}{c}{$\boldsymbol{\sigma}=\mathbf{2}$} & & \multicolumn{3}{c}{$\boldsymbol{\sigma}=\mathbf{0 . 1}$} \\
\cline { 2 - 4 } \cline { 6 - 7 } $\boldsymbol{t}$ & \multicolumn{1}{c}{$\boldsymbol{\tau}_{\boldsymbol{t}}^{\mathbf{1}}$} & $\boldsymbol{\tau}_{\boldsymbol{t}}^{2}$ & $\boldsymbol{b}_{\boldsymbol{t}}$ & & $\boldsymbol{\tau}_{\boldsymbol{t}}^{\mathbf{1}}$ & $\boldsymbol{\tau}_{\boldsymbol{t}}^{2}$ & $\boldsymbol{b}_{\boldsymbol{t}}$ \\
\hline 1 & +0.5 & -0.5 & +0.0 & & -4.9 & +4.9 & +0.0 \\
2 & +20.5 & -4.1 & -14.3 & & +0.1 & +13.5 & +9.5 \\
3 & +22.8 & -6.2 & -21.5 & & +1.3 & +12.4 & +4.6 \\
4 & +23.3 & -6.6 & -23.0 & & +2.1 & +11.8 & +1.5 \\
$\infty$ & +23.4 & -6.7 & -23.4 & +3.1 & +10.9 & -3.1 \\
\hline
\end{tabular}

Note: Percentage deviation from a constant $n$ solution. 
The short run, however, shows a different picture. When $\sigma$ is large, the adjustment is rapid and is realized essentially through a sharp decrease in national debt, or an increase in transfers to the young. Both policies aim at rapidly generating the newly required increased capital intensity: a decrease in public debt frees resources otherwise diverted from productive capital; part of the increase in the transfers to the young will end up as increased savings. The observed accompanying decrease in transfers to the old is another way to induce more savings by the young.

When $\sigma$ is small, the contrary is observed in the short run. Debt rises strongly in period 2 in order to allow for higher consumption levels, and a lower retirement age. The optimal lower retirement age, and the subsequent decrease in labor earnings of the old workers, together with the optimal increased consumption level of the old explain the considerable increase in transfers to the old.

According to these dynamic simulations we can thus conclude that, although the direction of the optimal response in terms of national debt and transfers to the young is insensitive to the concavity of the utility function in the long run, this is not necessarily so in the short run. More particularly, even when the optimal response to a drop in fertility in terms of public debt policy goes into the expected direction in the long run-i.e., public debt should decrease-this may not be the case during the transition.

\section{Conclusion}

In this paper, we focus on the consequences of a decline in fertility for the optimal allocation of resources. Most of the literature concerned with the aging of the population adopts a numerical approach and studies the effect of various policy reforms. Our objective is to switch the debate from the design of parametric reforms to the optimal allocation of resources (consumption, leisure, and capital). Endowed with the well-defined criterion of Ramsey (1928), we theoretically assess the impact of a fertility drop on the optimal allocation. In contrast with the theoretical contributions in this field, our model includes endogenous retirement decisions.

Our analysis of the long-run optimal allocation allows to conclude the following. First, the optimal capital intensity increases after a drop in population. Second, the optimal retirement age may either increase or decrease when the drop in fertility constitutes a positive shock for the economy. While it is then feasible and optimal to increase the per capita consumption of at least the young, the optimal response of the retirement age, however, crucially depends on the parameters, and in particular on the concavity of the utility function. The policy recommendation of postponing retirement is thus not robust to a wide class of preferences and technologies.

In order to translate these effects in terms of optimal retirement policies, we characterize the decentralization of the optimum either through lumpsum transfers or with public debt. In the long run, when the new optimal 
capital intensity and the consumption level of the young increases sufficiently, national debt should decrease or alternatively transfers to the young should increase.

The study of the optimal dynamics highlights that the welfare effects of the fertility drop on the initial generations also depend on the concavity of the utility function. As far as the decentralization instruments are concerned, dynamical simulations show that, even when the optimal public debt policy reaction goes into the expected direction in the long run, it may go into the opposite way during the transition.

\section{Appendix A: Proof of Proposition 1}

\section{A.1. Existence}

The steady state is characterized by the system of Equations (8)-(12).

On the one hand, the Golden Rule (11) determines a unique relationship in the space $\{k, \lambda\}$ between steady-state capital and $\lambda$ for given $n$ : when taking the total derivative of (11) we get $d k / d \lambda=-f_{k l} / f_{k k}>0$. On the other hand, Equations (8)-(12) establish another relationship between $k$ and $\lambda$ that should be verified at the steady state. We shall prove that both equations in $k$ and $\lambda$ intersect at least once, in which case we can conclude that there exists at least one steady state.

The Golden Rule (11) implies that $\forall \lambda \in(0,1)$, there exists one level of $k$ which satisfies this rule. Now define

$$
\begin{array}{lll}
\underline{k} \in \mathbb{R}_{+}: f_{k}(\underline{k}, n+\lambda)=n & \text { for } \quad \lambda=0 \\
\bar{k} \in \mathbb{R}_{+}: f_{k}(\bar{k}, n+\lambda)=n & \text { for } \quad \lambda=1 .
\end{array}
$$

with $\underline{k}<\bar{k}$. Here $\underline{k}$ and $\bar{k}$ are the levels of capital satisfying (11) for $\lambda=0$ and $\lambda=1$, respectively.

In the next step we identify two pairs $(k, \lambda)$ belonging to the curve defined by equations (8)-(12); one of which lies above and the other below the upward sloping $(\underline{k}, \bar{k})$-curve determined by (11). This will allow us to conclude that both curves cross at least once.

We define $\hat{k}$ as the steady-state capital which satisfies (8)-(12) for $\lambda=1$. Since $v_{\lambda}(1)=\infty$, according to (10) $q$ also tends to infinity $\left(f_{l}\right.$ is strictly positive and finite by assumption). Given (8) and (9) this implies that consumption in both periods tends to zero (because of the limit condition (1)). Hence the resource constraint (12) becomes $f(\hat{k}, n+1)=n \hat{k}$; or

$$
\frac{f(\hat{k}, n+1)}{\hat{k}}=n>f_{k}(\hat{k}, n+1) .
$$

The inequality derives from the Euler theorem implying that $f(k) / k>f_{k}$. Given (A1) and because of decreasing marginal productivity of capital, we 
can deduce that $\hat{k}>\bar{k}$, and thus that $(\hat{k}, n+1)$ lies above the $(\underline{k}, \bar{k})$-curve determined by the Golden Rule.

It is further straightforward to see that when $k=\underline{k},(8)-(12)$ imply that $\lambda \in(0,1)$ : on one hand we have that $f_{l}(\underline{k}, \cdot)$ is strictly positive and finite. On the other hand $f(\underline{k}, \cdot)$ is strictly positive and finite and greater than $n \underline{k}$. Indeed, $f(\underline{k}, n) / \underline{k}>f_{k}(\underline{k}, n)=n$ implies $f(\underline{k}, n+\lambda)>n \underline{k}$, allowing for strictly positive and finite consumption $c$ and $d$. Consequently, $q$ is also strictly positive and finite, given (8) and (9). Further, according to (10), $v_{\lambda} \in$ $(0, \infty)$, which implies that $\lambda \in(0,1)$ for $k=\underline{k}$. We can conclude that $\forall \lambda \in$ $(0,1),(\underline{k}, \lambda)$ lies below the $(\underline{k}, \bar{k})$-curve determined by (11) since the latter is upward sloping and starts in $(\underline{k}, 0)$. We can thus conclude that given continuity of (11) and (12), there is at least one intersection in the $(k, \lambda)$-plane.

\section{A.2. Uniqueness}

We can characterize the first-best solution as follows: an optimal allocation and its supporting implicit price $\left(q_{t}\right)_{t \geq 0}$ are characterized by the two dynamic equations

$$
\begin{aligned}
k_{t+1} & =\frac{1}{n_{t}}\left[f\left(k_{t}, n_{t}+\lambda_{t}\right)-\mathcal{C}\left(\frac{q_{t}}{f_{k}\left(k_{t}, n_{t}+\lambda_{t}\right)}\right)\right] \\
q_{t+1} & =\frac{n_{t} q_{t}}{f_{k}\left(k_{t}, n_{t}+\lambda_{t}\right)},
\end{aligned}
$$

where total consumption $\mathcal{C}(\cdot)$ is given by

$$
\begin{aligned}
\mathcal{C}\left(\frac{q_{t}}{f_{k}\left(k_{t}, n_{t}+\lambda_{t}\right)}\right)= & n_{t} c_{t}+d_{t}=n_{t} u_{c}^{-1}\left(\frac{n_{t} q_{t}}{f_{k}\left(k_{t}, n_{t}+\lambda_{t}\right)}\right) \\
& +u_{c}^{-1}\left(\frac{q_{t}}{\beta f_{k}\left(k_{t}, n_{t}+\lambda_{t}\right)}\right),
\end{aligned}
$$

and the static equation

$$
v_{\lambda}\left(\lambda_{t}\right)-q_{t} \frac{f_{l}\left(k_{t}, n_{t}+\lambda_{t}\right)}{f_{k}\left(k_{t}, n_{t}+\lambda_{t}\right)} \equiv h\left(k_{t}, q_{t}, \lambda_{t}\right)=0 .
$$

The dynamics of the economy can be reduced to a system of two equations:

$$
\begin{aligned}
& k_{t+1}=\Phi\left(k_{t}, q_{t}\right) \\
& q_{t+1}=\Psi\left(k_{t}, q_{t}\right) .
\end{aligned}
$$

In Appendix J we show that the slope $d k / d q$ of the curves (A5) and (A6) evaluated at the steady state are negative and positive, respectively. In Section A we showed that there exists at least one steady state. Hence both curves cross at least once. Suppose there is a second steady state implying that (A5) and (A6) cross again with a positive and negative slope, respectively. By continuity 
of (A5) and (A6) this would imply the existence of at least a third steady state, again determined by the intersection of the two curves, but where at least one of the curves has its slope reversed. This is in contradiction with the characterization of the steady state itself. Hence, the steady state is unique.

\section{A.3. Stability}

To study the characteristics of the dynamics, we take a first-order Taylor expansion of the system around its unique steady state $(k, q)$ in order to study the local dynamics. This leads to

$$
\left[\begin{array}{l}
k_{t+1}-k \\
q_{t+1}-q
\end{array}\right]=\left[\begin{array}{ll}
a_{1} & a_{2} \\
b_{1} & b_{2}
\end{array}\right]\left[\begin{array}{l}
k_{t}-k \\
q_{t}-q
\end{array}\right],
$$

with the partial derivatives taken at the steady state $(k, q)$ :

$$
\begin{aligned}
& a_{1}=\frac{\partial \Phi}{\partial k_{t}}=\frac{f_{k}}{n}+\frac{f_{l}}{n} \frac{d \lambda}{d k}+\frac{C_{z}}{n} \frac{q f_{k k}}{\left(f_{k}\right)^{2}}+\frac{C_{z}}{n} \frac{q f_{k l}}{\left(f_{k}\right)^{2}} \frac{d \lambda}{d k} \\
& a_{2}=\frac{\partial \Phi}{\partial q_{t}}=\frac{1}{n}\left(f_{l} \frac{d \lambda}{d q}-\frac{C_{z}}{f_{k}}+C_{z} \frac{q f_{k l}}{\left(f_{k}\right)^{2}} \frac{d \lambda}{d q}\right) \\
& b_{1}=\frac{\partial \Psi}{\partial k_{t}}=-n \frac{q}{\left(f_{k}\right)^{2}}\left(f_{k k}+f_{k l} \frac{d \lambda}{d k}\right) \\
& b_{2}=\frac{\partial \Psi}{\partial q_{t}}=\frac{n}{f_{k}}\left(1-\frac{q f_{k l}}{f_{k}} \frac{d \lambda}{d q}\right),
\end{aligned}
$$

where $C_{z}$ is the derivative of $C$ with respect to its argument. The derivatives $d \lambda / d k$ and $d \lambda / d q$ are given by

$$
\begin{aligned}
& \frac{d \lambda}{d k}=\frac{q f_{f l}-v_{\lambda} f_{k l}}{v_{\lambda \lambda} f_{k}+v_{\lambda} f_{k l}-q f_{l l}}>0 \\
& \frac{d \lambda}{d q}=\frac{f_{l}}{v_{\lambda \lambda} f_{k}+v_{\lambda} f_{k l}-q f_{l l}}>0 .
\end{aligned}
$$

The characteristic polynomial of the linear approximation is given by

$$
p(\mu)=\mu^{2}-\left(a_{1}+b_{2}\right) \mu+a_{1} b_{2}-a_{2} b_{1} .
$$

When substituting for $d \lambda / d k$ and $d \lambda / d q$, and rearranging terms we have that

$$
\begin{aligned}
a_{1}+b_{2} & =2+\frac{C_{z} q f_{k} f_{k k} v_{\lambda \lambda}-f_{l}\left(f_{k}\right)^{2} f_{k k} v_{\lambda}}{n\left(f_{k}\right)^{2}\left(v_{\lambda \lambda} f_{k}+v_{\lambda} f_{k l}-q f_{l l}\right)} \\
a_{1} b_{2}-a_{2} b_{1} & =1 .
\end{aligned}
$$

From (A7)-(A9) we can see that $p(0)>0$ and $p(1)<0$, which corresponds to a steady-state stable in the saddle-point sense. 


\section{Appendix B: Proof of Proposition 2}

To study the effect of a drop in $n$ on the optimal capital intensity we use $\tilde{k}$ and $\tilde{y}$, the capital and output per labor unit, respectively. So we have

$$
\tilde{k}=\frac{k}{n+\lambda} \quad \text { and } \quad \tilde{y}=\frac{y}{n+\lambda}=f(\tilde{k}),
$$

and the corresponding Golden Rule $f_{\tilde{k}}(\tilde{k})=n$. When deriving the Golden Rule with respect to $n$ it is straightforward to show that

$$
\frac{d \tilde{k}}{d n}=\frac{1}{f_{\tilde{k} \tilde{k}}}<0
$$

In order to study the effect of a drop in fertility on the optimal capital per old worker at the steady state, we compute the total derivatives of the optimality conditions (8)-(11) and the resource constraint (12) with respect to $c, d, k, \lambda, q$, and $n$. The total derivatives of (8) and (9) allow us to express $d c / c$ and $d d / d$ as follows:

$$
\begin{gathered}
\frac{d c}{c}=-\sigma(c) \frac{d q}{q} \quad \text { and } \quad \frac{d d}{d}=-\sigma(d)\left(\frac{d q}{q}-\frac{d n}{n}\right), \\
\text { where } \sigma(c)=-\frac{u_{c}(c)}{u_{c c}(c) c} .
\end{gathered}
$$

The term $\sigma(c)$ is the elasticity of substitution between consumption at two points of time. We also define

$$
v \equiv \frac{v_{\lambda \lambda} \lambda}{v_{\lambda}}>0
$$

as the elasticity of the disutility of work, and

$$
\eta \equiv \frac{f_{l l} l}{f_{l}}<0, \quad \epsilon \equiv \frac{f_{k l} l}{f_{k}}>0
$$

as the elasticities of the marginal productivity of labor and capital with respect to labor.

When substituting (B1) in the total derivatives of (10)-(12), we obtain a system of three equations and three unknowns, $d k / d n, d q / d n$, and $d \lambda / d n$. Using (B2) and (B3), we obtain

$$
\frac{d k}{d n} \frac{n}{k}=-\frac{1}{\epsilon}+\frac{c+k-c \sigma(c)+\left(\frac{\eta}{\epsilon}+\frac{v}{\lambda}\right)(n c \sigma(c)+d \sigma(d))}{c+\frac{d}{n}+v\left(\frac{1}{\lambda}+\frac{1}{n}\right)(n c \sigma(c)+d \sigma(d))} .
$$

This expression can be rewritten as

$$
\frac{-c \sigma(c)+\frac{\eta}{\epsilon}(n c \sigma(c)+d \sigma(d))}{N}+\frac{c+k-\frac{n}{n+\lambda}\left(c+\frac{d}{n}\right)}{N}+\frac{n}{n+\lambda}-\frac{1}{\epsilon^{\prime}},
$$


where $N=c+d / n+v(n+\lambda)(n c \sigma(c)+d \sigma(d)) /(n \lambda)>0$. The first term in (B5) is unambiguously negative. The second, third, and fourth term can be grouped as follows:

$$
\frac{c+k-\frac{n}{n+\lambda}\left(c+\frac{d}{n}\right)+\left(\frac{n}{n+\lambda}-\frac{1}{\epsilon}\right) N}{N} .
$$

When developing the numerator and after simplifying, (B6) becomes

$$
\frac{\nu\left(1-\frac{n+\lambda}{n \epsilon}\right) \frac{n c \sigma(c)+d \sigma(d)}{\lambda}}{N}+\frac{c+k-\frac{1}{\epsilon}\left(c+\frac{d}{n}\right)}{N} .
$$

In the case of a CES production function we can rewrite $1-(n+\lambda) /(n \epsilon)$ as $\left(n f_{l}-f /(1+\rho)\right) /\left(n f_{l}\right)$; it is straightforward to see that the first term in (B7) is negative for $\rho<0$. The numerator of the second term is equivalent to $c+k-f /(1+\rho)$, which is also clearly negative for $\rho<0$.

\section{Appendix C: Proof of Corollary 1}

When $\sigma$ tends to zero, (B4) becomes

$$
-\frac{1}{\epsilon}+\frac{c+k}{c+\frac{d}{n}}=\frac{c+k-\frac{1}{\epsilon}\left(c+\frac{d}{n}\right)}{c+\frac{d}{n}} .
$$

In the case of a CES production function, $c+k-1 / \epsilon(c+d / n)$ can be rewritten as $c+k-f /(1+\rho)$. This term will be positive for large $\rho$.

\section{Appendix D: Proof of Proposition 3}

By totally differentiating the resources constraint and using (B1), we get

$$
\left(c \sigma(c)+d \sigma(d) \frac{1}{n}\right) \frac{d q / q}{d n / n}=k+c-f_{l}+\frac{d \sigma(d)}{n}-\frac{d \lambda}{d n} f_{l} .
$$

When substituting (B4) into (13), the effect of a change in $n$ on $\lambda$ can be rewritten as

$$
\frac{d \lambda}{d n}=\frac{c+k-f_{l}-c \sigma(c)+(n c \sigma(c)+d \sigma(d)) \frac{\eta}{\epsilon}}{f_{l}+\frac{\nu(n c \sigma(c)+d \sigma(d))}{\lambda}} .
$$

This allows us to express $d \lambda / d n f_{l}$ as follows:

$$
\frac{c+k-f_{l}-X}{1+Y}
$$

with

$$
X=c \sigma(c)-(n c \sigma(c)+d \sigma(d)) \frac{\eta}{\epsilon}>0
$$


and

$$
Y=\frac{\nu(n c \sigma(c)+d \sigma(d))}{\lambda f_{l}}>0 .
$$

Equation (D1) therefore becomes

$$
\left(c \sigma(c)+d \sigma(d) \frac{1}{n}\right) \frac{d q / q}{d n / n}=\left(k+c-f_{l}\right)\left(1-\frac{1}{1+Y}\right)+\frac{d \sigma(d)}{n}+\frac{X}{1+Y} .
$$

It is straightforward to see that a sufficient condition for $\frac{d q}{d n}$ to be positive is that $\left(k+c-f_{l}\right)>0$.

\section{Appendix E: Proof of Corollary 2}

$$
\text { E.1. } \frac{d \lambda}{d n}>0 \Rightarrow \frac{d d}{d n}<0
$$

On one hand, when totally differentiating (9) with respect to $n$, we obtain

$$
\frac{d d}{d n} \frac{n}{d}=\sigma(d)\left(1-\frac{d q}{d n} \frac{n}{q}\right)
$$

On the other hand, totally differentiating (10) and (11) with respect to $n$ allows us to express $d q / d n$ as follows:

$$
\frac{d q}{d n} \frac{n}{q}=1-\frac{n}{f_{l}} \frac{f_{k l}}{f_{k k}}+\frac{n^{2}}{q} \frac{v_{\lambda \lambda}}{f_{l}} \frac{d \lambda}{d n}
$$

When substituting the above expression in (E1) we get

$$
\frac{d d}{d n} \frac{n}{d}=\sigma(d)\left(\frac{n}{f_{l}} \frac{f_{k l}}{f_{k k}}-\frac{n^{2}}{q} \frac{v_{\lambda \lambda}}{f_{l}} \frac{d \lambda}{d n}\right) .
$$

From (E2) it is straightforward to see that a decrease in the retirement age $(d \lambda / d n>0)$ implies an increase in consumption of the old.

$$
\text { E.2. } \frac{d d}{d n}<0 \Rightarrow \frac{d c}{d n}<0
$$

This result derives directly from Proposition 3.

\section{Appendix F: Proof of Corollaries 3 and 4}

From (D2) it is straightforward to see that $d \lambda / d n<0$ (a) for large $\sigma$, and (b) when $f_{l}-c-k>0$.

\section{Appendix G: Proof of Proposition 5}

We follow the proof in de la Croix and Michel (2002). The government budget constraint and Equation (15) allow us to express the transfers in the following 
way, for all $t \geq 0$ :

$$
\tau_{t}^{2}=d_{t}^{\star}-f_{k}\left(k_{t}^{\star}, n_{t}+\lambda_{t}^{\star}\right) k_{t}^{\star}-f_{l}\left(k_{t}^{\star}, n_{t}+\lambda_{t}^{\star}\right) \lambda_{t}^{\star},
$$

which are the levels that allow the old workers to consume $d_{t}^{\star}$ at equilibrium. Using the resource constraint and the Euler theorem, this implies

$$
\tau_{t}^{2}=n_{t}\left(f_{l}\left(k_{t}^{\star}, n_{t}+\lambda_{t}^{\star}\right)-k_{t+1}^{\star}-c_{t}^{\star}\right) .
$$

The corresponding transfer to the young is given by

$$
\tau_{t}^{1}=k_{t+1}^{\star}+c_{t}^{\star}-f_{l}\left(k_{t}^{\star}, n_{t}+\lambda_{t}^{\star}\right) .
$$

Consider any date $t \geq 0$. At the given capital stock $k_{t}^{\star}$, and assuming perfect forecasts, the optimal choices $c_{t}, d_{t+1}$, and $s_{t}$ of the agents for period $t$ are characterized by

$$
\begin{aligned}
u_{c}\left(c_{t}\right) & =f_{k}\left(k_{t+1}^{\star}, n_{t+1}+\lambda_{t+1}\right) \beta u_{c}\left(d_{t+1}\right) \\
v_{\lambda}\left(\lambda_{t+1}\right) & =\beta u_{c}\left(d_{t+1}\right) f_{l}\left(k_{t+1}^{\star}, n_{t+1}+\lambda_{t+1}\right) \\
c_{t} & =f_{l}\left(k_{t}^{\star}, n_{t}+\lambda_{t}\right)+\tau_{t}^{1}-s_{t} \\
d_{t+1} & =f_{k}\left(k_{t+1}^{\star}, n_{t+1}+\lambda_{t+1}\right) s_{t}+\tau_{t+1}^{2}+f_{l}\left(k_{t+1}^{\star}, n_{t+1}+\lambda_{t+1}\right) \lambda_{t+1} .
\end{aligned}
$$

From Equations (9) and (10) we know that the optimal retirement age is characterized by

$$
\beta u_{c}\left(d_{t+1}\right)=\frac{v_{\lambda}\left(\lambda_{t+1}^{\star}\right)}{f_{l}\left(k_{t+1}^{\star}, n_{t+1}+\lambda_{t+1}^{\star}\right)},
$$

which implies that $\lambda_{t+1}=\lambda_{t+1}^{\star}$.

After substituting for the transfers, the last two equations become

$$
\begin{aligned}
c_{t}= & k_{t+1}^{\star}+c_{t}^{\star}-s_{t} \\
d_{t+1}= & f_{k}\left(k_{t+1}^{\star}, n_{t+1}+\lambda_{t+1}^{\star}\right) s_{t}+\left(n_{t+1}+\lambda_{t+1}^{\star}\right) f_{l}\left(k_{t+1}^{\star}, n_{t+1}+\lambda_{t+1}^{\star}\right) \\
& -n_{t+1}\left(k_{t+2}^{\star}+c_{t+1}^{\star}\right) \\
= & f_{k}\left(k_{t+1}^{\star}, n_{t+1}+\lambda_{t+1}^{\star}\right)\left(s_{t}-k_{t+1}^{\star}\right)+f\left(k_{t+1}^{\star}, n_{t+1}+\lambda_{t+1}^{\star}\right) \\
& -n_{t+1}\left(k_{t+2}^{\star}+c_{t+1}^{\star}\right) \\
= & f_{k}\left(k_{t+1}^{\star}, n_{t+1}+\lambda_{t+1}^{\star}\right)\left(s_{t}-k_{t+1}^{\star}\right)+d_{t+1}^{\star},
\end{aligned}
$$

where $s_{t}=k_{t+1}^{\star}, \lambda_{t+1}=\lambda_{t+1}^{\star}, c_{t}=c_{t}^{\star}$, and $d_{t+1}=d_{t+1}^{\star}$ is the unique solution of the above system. 


\section{Appendix H: Proof of Proposition 6}

Using (22), the government budget constraint (24), and the capital market equilibrium condition (25), the level of public debt that allows the young agents to consume $c_{t}^{\star}$ at equilibrium can be defined as follows:

$$
c_{t}^{\star}=f_{l}\left(k_{t}^{\star}, n_{t}+\lambda_{t}^{\star}\right)+\tau_{t}-k_{t+1}^{\star}-b_{t+1}
$$

or equivalently as

$$
b_{t+1}=f_{l}\left(k_{t}^{\star}, n_{t}+\lambda_{t}^{\star}\right)+\tau_{t}-k_{t+1}^{\star}-c_{t}^{\star} .
$$

From the government budget constraint, the corresponding transfer level is

$$
\tau_{t}=\frac{n_{t} b_{t+1}-f_{k}\left(k_{t}^{\star}, n_{t}+\lambda_{t}^{\star}\right) b_{t}}{n_{t}} .
$$

Consider any date $t \geq 0$. At the given capital stock $k_{t}^{\star}$, and assuming perfect forecasts, the optimal choices $c_{t}, d_{t+1}$, and $s_{t}$ of the agents for period $t$ are characterized by

$$
\begin{aligned}
u_{c}\left(c_{t}\right) & =f_{k}\left(k_{t+1}^{\star}, n_{t+1}+\lambda_{t+1}\right) \beta u_{c}\left(d_{t+1}\right) \\
v_{\lambda}\left(\lambda_{t+1}\right) & =\beta u_{c}\left(d_{t+1}\right) f_{l}\left(k_{t+1}^{\star}, n_{t+1}+\lambda_{t+1}\right) \\
c_{t} & =f_{l}\left(k_{t}^{\star}, n_{t}+\lambda_{t}\right)+\tau_{t}-s_{t} \\
d_{t+1} & =f_{k}\left(k_{t+1}^{\star}, n_{t+1}+\lambda_{t+1}\right) s_{t}+f_{l}\left(k_{t+1}^{\star}, n_{t+1}+\lambda_{t+1}\right) \lambda_{t+1} .
\end{aligned}
$$

From Equations (9) and (10) we know that the optimal retirement age is characterized by

$$
\beta u_{c}\left(d_{t+1}\right)=\frac{v_{\lambda}\left(\lambda_{t+1}^{\star}\right)}{f_{l}\left(k_{t+1}^{\star}, n_{t+1}+\lambda_{t+1}^{\star}\right)},
$$

which implies that $\lambda_{t+1}=\lambda_{t+1}^{\star}$. After substituting for the transfers and using (25), Equation (H1) becomes

$$
c_{t}=c_{t}^{\star} \text {. }
$$

Substituting for the transfers in Equation (H2), we get

$$
\begin{aligned}
d_{t+1}= & f_{k}\left(k_{t+1}^{\star}, n_{t+1}+\lambda_{t+1}^{\star}\right) k_{t+1}^{\star}+f_{l}\left(k_{t+1}^{\star}, n_{t+1}+\lambda_{t+1}^{\star}\right) \lambda_{t+1}^{\star} \\
& +f_{k}\left(k_{t+1}^{\star}, n_{t+1}+\lambda_{t+1}^{\star}\right) b_{t+1} \\
= & f\left(k_{t+1}^{\star}, n_{t+1}+\lambda_{t+1}^{\star}\right)-f_{l}\left(k_{t+1}^{\star}, n_{t+1}+\lambda_{t+1}^{\star}\right) n_{t+1}+n_{t+1}\left(b_{t+2}-\tau_{t+1}\right) \\
= & d_{t+1}^{\star}+n_{t+1}\left(c_{t+1}^{\star}+k_{t+2}^{\star}-f_{l}\left(k_{t+1}^{\star}, n_{t+1}+\lambda_{t+1}^{\star}\right)\right) \\
& +n_{t+1}\left(-c_{t+1}^{\star}-k_{t+2}^{\star}+f_{l}\left(k_{t+1}^{\star}, n_{t+1}+\lambda_{t+1}^{\star}\right)\right) \\
= & d_{t+1}^{\star} .
\end{aligned}
$$


Therefore, $b_{t+1}+s_{t}=k_{t+1}^{\star}, c_{t}=c_{t}^{\star}, d_{t+1}=d_{t+1}^{\star}$, and $\lambda_{t+1}=\lambda_{t+1}^{\star}$ is the unique solution of the above system.

\section{Appendix I: Proof of Proposition 7}

The steady-state level of transfer to the young is given by $\tau^{1}=-\left(f_{l}(k, n+\lambda)-\right.$ $k-c)$. By totally differentiating this expression we obtain:

$$
\frac{d \tau^{1}}{d n}=-\left(f_{l k}(k, n+\lambda) \frac{d k}{d n}+f_{l l}(k, n+\lambda)\left(1+\frac{d \lambda}{d n}\right)-\frac{d c}{d n}-\frac{d k}{d n}\right) .
$$

Using (13) we get

$$
\begin{aligned}
\frac{d \tau^{1}}{d n} & =-\left(\left(f_{k l}(k, n+\lambda)-1+f_{l l}(k, n+\lambda) \frac{n+\lambda}{k}\right) \frac{d k}{d n} \frac{f_{l l}(k, n+\lambda)}{f_{k l}(k, n+\lambda)}-\frac{d c}{d n}\right) \\
& =-\left(-\frac{d k}{d n}-\frac{d c}{d n}-\frac{k}{n+\lambda}\right) \\
& =-\frac{k}{n}\left(-\frac{n}{k} \frac{d k}{d n}+\sigma(c) \frac{d q}{d n} \frac{n}{q} \frac{c}{k}-\frac{n}{n+\lambda}\right) .
\end{aligned}
$$

At the steady state, the public debt is given by $b=f_{l}(k, n+\lambda)-c-k=-\tau^{1}$. The total differentiation of this equation is therefore given by

$$
\frac{d b}{d n}=-\frac{d \tau^{1}}{d n}=\frac{k}{n}\left(-\frac{n}{k} \frac{d k}{d n}+\sigma(c) \frac{d q}{d n} \frac{n}{q} \frac{c}{k}-\frac{n}{n+\lambda}\right) .
$$

\section{Appendix J: The Phase Diagram}

To build this diagram we use the two equations of the dynamic system (28) and (29):

$$
\begin{aligned}
& k_{t+1}=\Phi\left(k_{t}, q_{t}, \lambda_{t}\right) \\
& q_{t+1}=\Psi\left(k_{t}, q_{t}, \lambda_{t}\right),
\end{aligned}
$$

where $\lambda_{t}$ is defined by a function $\Omega$ applying the implicit function theorem to $(\mathrm{A} 4)$ :

$$
\lambda_{t}=\Omega\left(k_{t}, q_{t}\right) \Leftrightarrow h\left(k_{t}, q_{t}, \lambda_{t}\right)=0 .
$$

The set of points $\left(k_{t}, q_{t}\right)$ for which there is no change in $k_{t}$ is characterized by $k_{t}=\Phi\left(k_{t}, q_{t}, \Omega\left(k_{t}, q_{t}\right)\right)$. By totally differentiating this equation, one can show that this phase line is downwards sloping around the steady state. This result is proven in Section J.1. To describe the direction of a change in $k_{t}$, we notice that $\Phi(\cdot)$ increases monotonically with $q_{t}$. Hence, $k_{t+1}>k_{t}$ above the curve and $k_{t+1}<k_{t}$ below. The set of points $\left(k_{t}, q_{t}\right)$ for which there is no 
change in $q_{t}$ is characterized by $q_{t}=\Psi\left(k_{t}, q_{t}, \Omega\left(k_{t}, q_{t}\right)\right)$. At the steady state the slope of this phaseline is positive. This is shown in Section J.2. To describe the direction of a change in $q_{t}$, we notice that $\Psi(\cdot)$ increases unambiguously with $k_{t}$. Hence, $q_{t+1}<q_{t}$ at the left of the phaseline and $q_{t+1}>q_{t}$ at the right of it.

\section{J.1. The Slope of the Phaseline $\Phi\left(k_{t}, q_{t}\right)=k_{t}$}

The slope of this curve is obtained by totally differentiating this expression at the steady state:

$$
\frac{d q}{d k}=-\frac{\partial \Phi / \partial k}{\partial \Phi / \partial q}=-\frac{\Phi_{k}+\Phi_{\lambda} \Omega_{k}-1}{\Phi_{q}+\Phi_{\lambda} \Omega_{q}}
$$

with

$$
\begin{aligned}
& \Phi_{k}=1+\frac{q}{n^{3}} f_{k k} C_{z}>0 \\
& \Phi_{q}=-\frac{C_{z}}{n^{2}}>0 \\
& \Phi_{\lambda}=\frac{f_{l}}{n}+\frac{q}{n^{3}} f_{k l} C_{z} \\
& \Omega_{q}=-\frac{h_{q}}{h_{\lambda}}=\frac{f_{l}}{v_{\lambda \lambda} f_{k}+v_{\lambda} f_{k l}-q f_{l l}}=\frac{-f_{l} f_{k}}{v_{\lambda \lambda} f_{k}^{2}+q f_{l} f_{k l}-q f_{k} f_{l l}}>0 \\
& \Omega_{k}=-\frac{h_{k}}{h_{\lambda}}=\frac{-q f_{k} f_{k k}+q f_{k} f_{k l}}{v_{\lambda \lambda} f_{k}+v_{\lambda} f_{k l}-q f_{l l}}=\frac{-q f_{k} f_{l l}}{v_{\lambda \lambda} f_{k}^{2}+q f_{k l} f_{l}-q,}
\end{aligned}
$$

where we substituted $v_{\lambda}=\frac{q f_{l}}{f_{k}}$. Let us show that the numerator of $(\mathrm{J} 1)$ is positive:

$$
\begin{aligned}
\frac{\partial \Phi}{\partial k}=\Phi_{k}+\Phi_{\lambda} \Omega_{k}-1 & =\frac{f_{l}}{n} \Omega_{k}+\frac{q}{n^{3}} f_{k k} C_{z}+\frac{q}{n^{3}} f_{k l} C_{z} \Omega_{k} \\
& =\frac{f_{l}}{n} \Omega_{k}+\frac{q C_{z}}{n^{3}}\left(f_{k k}+f_{k l} \Omega_{k}\right) .
\end{aligned}
$$

The first term is positive. When substituting for $\Omega_{k}$ in the second term it is straightforward to show that $f_{k k}+f_{k l} \Omega_{k}$ is negative, which implies that the second term is also positive (with $C_{z}<0$ ). Consequently, the numerator is positive.

The denominator of (J1)

$$
\frac{\partial \Phi}{\partial q}=\Phi_{q}+\Phi_{\lambda} \Omega_{q}=\frac{f_{l} \Omega_{q}}{n}+\frac{C_{z}}{n^{2}}\left(-1+\frac{q}{n} f_{k l} \Omega_{q}\right)
$$

can also proven to be positive. The first term is positive. After substituting for $\Omega_{q}$ it is again straightforward to show that the second term is also positive. The 
denominator is therefore positive. We can thus conclude that (J1) is negative which implies that the phaseline $\Phi\left(k_{t}, q_{t}\right)=k_{t}$ is downward sloping.

\section{J.2. The Slope of the Phaseline $\Psi\left(k_{t}, q_{t}\right)=q_{t}$}

The slope of this phaseline is obtained by totally differentiating the equation at the steady state:

$$
\frac{d q}{d k}=-\frac{\partial \Psi / \partial k}{\partial \Psi / \partial q}=-\frac{\Psi_{k}+\Psi_{\lambda} \Omega_{k}}{\Psi_{q}+\Psi_{\lambda} \Omega_{q}-1}
$$

with

$$
\begin{aligned}
& \Psi_{k}=-\frac{q f_{k k}}{n}>0 \\
& \Psi_{q}=1 \\
& \Psi_{\lambda}=-\frac{q f_{k l}}{n}<0 .
\end{aligned}
$$

The sign of the numerator $\partial \Psi / \partial k$ can easily be proven to be positive given that $\left(f_{k k}+f_{k l} \Omega_{k}\right)<0$ which implies $\Psi_{k}+\Psi_{\lambda} \Omega_{k}>0$. Since $\Omega_{q}>0$ and $\Psi_{\lambda}<0$, it is straightforward to show that the denominator $\partial \Psi / \partial q$ is negative. Consequently, the phaseline $\Psi\left(k_{t}, q_{t}\right)=q_{t}$ is upward sloping.

\section{References}

ATKINSON, A., and A. SANDMO (1980) Welfare implications of the taxation of savings, Economic Journal 90, 529-549.

AUERBACH, A., L. KOTLIKOFF, R. HAGEMANN, and G. NICOLETTI (1989) The economic dynamics of an ageing population: The case of four OECD countries, Working Paper 62, OECD.

BLANCHET, D., and D. KESSLER (1991) Optimal pension funding with demographic instability and endogenous returns on investment, Journal of Population Economics 4, 137-154.

BOADWAY, R., M. MARCHAND, and P. PESTIEAU (1991) Pay-as-you-go social security in a changing environment, Journal of Population Economics 4, 257-280.

CAZES, S., T. CHAUVEAU, J. LE CACHEUX, and R. LOUFIR (1994) Public pensions in an overlapping generations model of the French economy, Keio Economic Studies 31, 1-19.

CHAUVEAU, T., and R. LOUFIR (1996) The future of public pensions in Japan, Keio Economic Studies 33, 71-92.

CONESA, J., and D. KRUEGER (1999) Social security reform with heterogeneous agents, Review of Economic Dynamics 2, 757-795. 
CRETTEZ, B., and P. LE MAITRE (2002) Optimal age of retirement and population growth, Journal of Population Economics 15 737-755.

CUTLER, D., J. POTERBA, L. SHEINER, and L. SUMMERS (1990) An aging society: Opportunity or challenge?, Brookings Papers on Economic Activity, 1-56.

DE LA CROIX, D., and P. MICHEL (2002) A Theory of Economic Growth: Dynamics and Policy in Overlapping Generations. Cambridge: Cambridge University Press.

DE NARDI, M., S. IMROHOROGLU, and T. SARGENT (1999) Projected U.S. demographics and social security, Review of Economic Dynamics 2, 575-615.

DEARDORFF, A. (1976) The optimum growth rate of population: Comment, International Economic Review 17, 510-515.

DIAMOND, P. (1965) National debt in a neoclassical growth model, American Economic Review 55, 1126-1150.

HU, S. (1979) Social security, the supply of labor, and capital accumulation, American Economic Review 69, 274-283.

HUANG, H., S. IMROHOROGLU, and T. SARGENT (1997) Two computations to fund social security, Macroeconomic Dynamics 1, 7-44.

KOTLIKOFF, L. (1996) Simulating the privatization of social security in general equilbrium, Working Paper 5776, NBER.

KOTLIKOFF, L., K. SMETTERS, and J. WALLISER (2001) Finding a way out of America's demographic dilemma, Working Paper 8258, NBER.

MARCHAND, M., P. MICHEL, and P. PESTIEAU (1996) Intergenerational transfers in an endogenous growth model with fertility changes, European Journal of Political Economy 12, 33-48.

MEIJDAM, L., and H. VERBON (1997) Aging and public pensions in an overlapping generations model, Oxford Economic Papers 49, $29-42$.

MICHEL, P. (1990a) Some clarifications on the transversality conditions, Econometrica 58, 705-723.

MICHEL, P. (1990b) Criticism of the social time-preference hypothesis in optimal growth, Working Paper 9039, CORE, Université catholique de Louvain.

MICHEL, P., and P. PESTIEAU (1999) Social security and early retirement in an overlapping generations growth model, Working Paper 9951, CORE, Université catholique de Louvain.

MILES, D. 1999. Modelling the impact of demographic change upon the economy, Economic Journal 109, 1-36.

PETERS, W. (1991) Public pensions in transition. An optimal policy path, Journal of Population Economics 4, 155-176.

RAMSEY, F. (1928) A mathematical theory of savings, Economic Journal 38, 543-559.

RUST, J., and C. PHELAN (1997) How social security and medicare affect retirement behavior in a world of incomplete markets, Econometrica 65, 781-831. 
SAMUELSON, P. (1975) The optimum growth rate of population, International Economic Review 16, 531-537.

SAMUELSON, P. (1976) The optimum growth rate for population: Agreement and evaluations, International Economic Review 17, 516-525.

SOLOW, R. (1974) Intergenerational equity and exhaustible resources, Review of Economic Studies 41, 29-45. 